\title{
Quantum Integrable 1D anyonic Models: Construction through Braided Yang-Baxter Equation ${ }^{\star}$
}

\author{
Anjan KUNDU
}

Theory Group 83 CAMCS, Saha Institute of Nuclear Physics, Calcutta, India

E-mail: anjan.kundu@saha.ac.in

URL: http://www.saha.ac.in/theory/anjan.kundu/

Received May 25, 2010, in final form October 03, 2010; Published online October 09, 2010

doi:10.3842/SIGMA.2010.080

\begin{abstract}
Applying braided Yang-Baxter equation quantum integrable and Bethe ansatz solvable 1D anyonic lattice and field models are constructed. Along with known models we discover novel lattice anyonic and $q$-anyonic models as well as nonlinear Schrödinger equation (NLS) and the derivative NLS quantum field models involving anyonic operators, $N$-particle sectors of which yield the well known anyon gases, interacting through $\delta$ and derivative $\delta$-function potentials.
\end{abstract}

Key words: nonultralocal model; braided YBE; quantum integrability; 1D anyonic and $q$-anyonic lattice models; anyonic NLS and derivative NLS field models; algebraic Bethe ansatz

2010 Mathematics Subject Classification: 16T25; 20F36; 81R12

\section{Introduction}

Anyons [1] are receiving renewed attention after their experimental confirmation [2] and the promise of their potential applications in quantum computation [3]. Although the anyons live in two space-dimensions, they remarkably retain their basic properties when projected to onedimension (1D) [4]. Therefore, since exactly solvable models are possible to construct in 1D, interacting $1 \mathrm{D}$ anyonic models introduced in $[5,6,7]$ are becoming increasingly popular in recent years $[4,8]$, with experimental verification of $1 \mathrm{D}$ anyon realised just recently [9]. However, though these models capture the exchange algebra of anyonic operators at the space-separated points $x \neq y$, they behave like bosons [5, 6, 4, 8] or fermions [7] at the coinciding points, due to their inability to reproduce the required anyonic commutation relation $(\mathrm{CR})$ at $x=y$ and consequently the corresponding operators can not interpolate between bosonic and fermionic operators in the entire domain. To resolve this problem in the known $1 \mathrm{D}$ anyonic models remains therefore as a challenge.

Another major unsettled problem is that, unlike the nonlinear Schrödinger equation (NLS) and the derivative NLS, which give the known solvable Bose gases $[10,11]$ at their $N$-particle sectors, no anyonic quantum field models are discovered yet, which could yield the known anyon gases at the $N$-particle case, $[5,6]$.

Note however that the anyons, not commuting at space-separated points, belong to nonultralocal models and go beyond the standard formulation of the quantum inverse scattering method (ISM) [14] and therefore, for constructing quantum integrable anyonic models we have to use the nonultralocal extension of the ISM introduced in $[12,13]$. Our aim here is to resolve both the above mentioned problems in the present 1D anyonic models, by finding quantum integrable anyonic lattice and field models, based on the braided extension of the Yang-Baxter

\footnotetext{
${ }^{\star}$ This paper is a contribution to the Proceedings of the International Workshop "Recent Advances in Quantum Integrable Systems". The full collection is available at http://www.emis.de/journals/SIGMA/RAQIS2010.html
} 
equation (BYBE) $[12,13]$. However, we intend to announce here our preliminary results only, a detail account of which, focusing on all aspects of the present result as well as our other related findings, will be given elsewhere in a separate publication [15].

The arrangement of the paper is as follows. Section 2 describes briefly the introduction of 1D anyonic models. Section 3 presents the BYBE and the related quantum integrable nonultralocal models. Subsection 3.3 constructs the quantum integrable anyonic lattice and the anyonic NLS field models. Subsection 3.4 accounts for the $q$-anyonic and the derivative NLS anyonic field models. Section 4 is the concluding section followed by the bibliography.

\section{Exactly solvable 1D boson and anyon gases}

Anyons continue to exhibit nontrivial exchange and cross-over properties even when projected to $1 \mathrm{D}$, with the 2-particle anyonic wave function showing a mixed symmetry under exchange

$$
\Phi\left(x_{1}, x_{2}\right)=e^{-i \theta} \Phi\left(x_{2}, x_{1}\right),
$$

interpolating between symmetric and antisymmetric wave functions. Anyonic wave function also exhibit an intriguing sensitivity on the boundary condition:

$$
\Phi\left(x_{1}+L, x_{2}\right)=e^{-2 i \theta} \Phi\left(x_{1}, x_{2}+L\right),
$$

on a chain of length $L$ [4], confirming that the passing of particle 1 through 2 in $1 \mathrm{D}$ is not the same as particle 2 passing through 1 . This reflects the known property of the standard $2 \mathrm{D}$ anyon, where the effect of particle 1 going around 2 is different from that of 2 going around 1 . Note that from (1), (2) one recovers the usual bosonic behavior at $\theta=0$, while $\theta=\pi$ corresponds to the fermion. The focus on 1D anyon is intensified in recent years, since together with preserving the basic properties of the standard anyon, in 1D anyons can be constructed as exactly solvable models offering detailed analytic result, which should be valuable for analyzing the standard anyons.

Introduction of the exactly solvable anyonic models in 1D went through some exciting events with the active involvement of the present author. Solvable many particle bosonic models, interacting through singular potentials were known for long. The celebrated model of Bose gas with $\delta$-function potential was introduced way back in 1963 by Lieb \& Liniger [10]. After about thirty years another Bose gas model, interacting through derivative $\delta$-function potential was proposed [11]. Both these bosonic models were solved exactly by the Bethe ansatz. After such a success of Bose gases with singular potentials, there were naturally attempts to build other interacting bosonic models with higher singular potentials, like double $\delta$-function potentials of the form

$$
\gamma_{1} \sum_{\langle j, k, l\rangle} \delta\left(x_{j}-x_{k}\right) \delta\left(x_{l}-x_{k}\right)+\gamma_{2} \sum_{\langle k, l\rangle}\left(\delta\left(x_{k}-x_{l}\right)\right)^{2},
$$

involving 2- and 3-particle interactions. However such attempts remained unsuccessful until the introduction of $\delta$-function anyon gas by the present author [5], which is an exactly solvable $1 \mathrm{D}$ model and shown indeed to be equivalent to a double $\delta$-Bose gas involving higher singular potentials (3) with its coupling constants related by $\gamma_{1}=\gamma_{2}=\kappa^{2}$. This anyonic model demonstrated clearly, contrary to the prevailing belief, that the the Bethe ansatz is applicable beyond symmetric or antisymmetric wave functions.

Another exactly solvable 1D anyon gas model, interacting through derivative $\delta$-function potential, is also proposed recently [6], with the participation of the present author. When these anyonic gas models, with different research groups focusing on their various aspects, were gaining popularity [8], other interesting nearest neighbor lattice anyonic models, solvable by the 
algebraic Bethe ansatz, were proposed quite recently [7]. However, as mentioned, 1D anyonic models proposed so far suffer from the common drawback that, they behave like bosons or fermions at the coinciding points.

Another important well known fact is that, for every exactly solvable Bose gas model, there exists a quantum integrable bosonic field model, $N$-particle sector of which corresponds to the interacting Bose gas. In fact the nonlinear Schrödinger equation (NLS)

$$
H^{(b 1)}=\int d x\left(\psi_{x}^{\dagger} \psi_{x}+c\left(\psi^{\dagger} \psi\right)^{2}\right)
$$

in bosonic field $\left[\psi(x), \psi^{\dagger}(y)\right]=\delta(x-y)$ corresponds to the $\delta$-Bose gas, while the quantum integrable derivative NLS field model yields in the $N$-particle case the derivative $\delta$-function Bose gas. However an important question surrounding the 1D anyon gases, that remained unanswered up to this date is that, what are the quantum integrable anyonic field models, $N$-particle sectors of which could generate the known anyon gas models interacting through $\delta$ and derivative $\delta$-function potentials. Our aim here is to take up this challenging problem and discover the needed quantum integrable anyonic QFT models. We also intend to construct integrable anyonic lattice and field models in a systematic way through Yang-Baxter equation, so as to guarantee the anyonic commutation relations of its operators at all points, including the coinciding points, thus resolving an existing problem of the anyonic models mentioned above.

\section{Construction of integrable anyonic models through braided Yang-Baxter equation}

Anyonic models due to the non commutation of anyonic fields at space separated points belong to the class of nonultralocal models and go beyond the standard formulation of quantum integrable systems based on the YBE [14]. We have to use therefore an extension of the YBE with nontrivial braiding (BYBE) developed by us [12, 13], for systematic generation of the anyonic commutation relations as well as for the construction of quantum integrable anyonic models.

\subsection{Braided Yang-Baxter equation}

The BYBE represents two different commutation relations for the Lax operator $L_{a j}(u)$, given at the coinciding and noncoinciding points, expressed through the standard quantum $R(u-v)$ matrix in addition to a braiding matrix $Z$ :

$$
R_{12}(u-v) Z_{21}^{-1} L_{1 j}(u) Z_{21} L_{2 j}(v)=Z_{12}^{-1} L_{2 j}(v) Z_{12} L_{1 j}(u) R_{12}(u-v),
$$

at the lattice sites $j=1,2, \ldots, N$, together with the braiding relation $(\mathrm{BR})$ :

$$
L_{2 k}(v) Z_{21}^{-1} L_{1 j}(u)=Z_{21}^{-1} L_{1 j}(u) Z_{21} L_{2 k}(v) Z_{21}^{-1}
$$

for $k>j$, representing nonultralocality, i.e. noncommutativity at space separated points. Recall that the quantum $R(u-v)$ matrix is a $4 \times 4$ matrix

$$
R(\lambda)=\left(\begin{array}{cccc}
a(\lambda) & & & \\
& b(\lambda) & c & \\
& c & b(\lambda) & \\
& & & a(\lambda)
\end{array}\right)
$$


with rational

$$
a(\lambda)=\lambda+\alpha, \quad b(\lambda)=\lambda, \quad c=\alpha
$$

or trigonometric

$$
a(\lambda)=\sin (\lambda+\alpha), \quad b(\lambda)=\sin \lambda, \quad c=\sin \alpha
$$

solutions. We consider both of these forms and show that they would generate two different classes of anyonic integrable models. The braiding matrix $Z$ containing the anyonic parameter $\theta$, may be given in the graded form

$$
Z=\sum_{a, b} e^{i \theta(\hat{a} \cdot \hat{b})} e_{a, b} \otimes e_{b, a}, \quad \hat{a}=0,1 \text { denotes anyonic gradings }
$$

which satisfies all the relations as required for the braided generalization [12]. For a $4 \times 4$ matrix with the choice $\hat{1}=0, \hat{2}=1$ we get the simplest form

$$
Z=\operatorname{diag}\left(1,1,1, e^{i \theta}\right)
$$

which we use in constructing all our anyonic models. It is evident that for $\theta=0: Z=I$, BYBE (4) reduces to the standard YBE $R(u-v) L_{1 j}(u) L_{2 j}(v)=L_{2 j}(v) L_{1 j}(u) R(u-v)$, while the $\mathrm{BR}(5)$ recovers the ultralocal condition $\left[L_{2 k}(v), L_{1 j}(u)\right]=0, k \neq j$, related to the bosonic commutativity.

\subsection{Construction of quantum integrable models}

For building the Hamiltonian of the model we have to construct conserved quantities by switching over from the local to a global picture, by defining the transfer matrix as a global quantum operator acting on the multi-particle Hilbert space:

$$
\tau(u)=\operatorname{tr}_{a}\left(L_{a 1}(u) \cdots L_{a N}(u)\right),
$$

which generates all conserved quantities $\log \tau(u)=\sum_{n} C_{n} u^{n}$. The BYBE guarantees that $[\tau(u), \tau(v)]=0$, and hence the commutativity of the conserved operators $\left[C_{n}, C_{m}\right]=0$, ensuring the quantum integrability of the model. Hamiltonian of the model can be chosen as any of the conserved operators: $H=C_{n}, n=1,2,3, \ldots$, which can therefore be constructed via (10) from the Lax operator $L_{j}(u)$, satisfying the BYBE (4) with the known solution of the quantum $R$-matrix and the braiding matrix $Z$.

\subsection{Rational class of anyonic models}

Let us consider first the rational $R$-matrix solution (6), (7) together with the $Z$-matrix (9), by taking the Lax operator in a general form

$$
L_{a(l)}^{b}(\lambda)=\lambda \delta_{a b} p_{b}^{0(l)}+\alpha p_{b a}^{(l)},
$$

involving abstract anyonic operators $\mathbf{p} \equiv\left(p_{b a}^{(l)}, p_{b}^{0(l)}\right), a, b=1,2$. The anyonic commutation relations $(\mathrm{CR})$ are obtained directly from the BYBE and the BR as

$$
\begin{aligned}
& p_{12}^{(l)} p_{21}^{(l)}-s^{-1} p_{21}^{(l)} p_{12}^{(l)}=p_{11}^{(l)} p_{2}^{0(l)}-s^{-1} p_{1}^{0(l)}, \\
& p_{12}^{(k)} p_{12}^{(j)}=s^{-1} p_{12}^{(j)} p_{12}^{(k)}, \quad k>j .
\end{aligned}
$$


Different realizations of these operators would generate different anyonic models with the corresponding anyonic algebra, presented below. Detailed account of these models will be given elsewhere [15].

i) Lattice hard-core anyonic model. Implementing the above described scheme for the construction of lattice anyonic models and realizing the general $\mathbf{p}$ operator as

$$
\begin{aligned}
& p_{12}^{(k)}=a_{k}^{\dagger}, \quad p_{21}^{(k)}=a_{k}, \quad p_{11}^{(k)}=n_{k}, \quad p_{22}^{(k)}=1-n_{k}, \\
& p_{1}^{0(k)}=1+(s-1) n_{k}, \quad n_{k}=a_{k}^{\dagger} a_{k},
\end{aligned}
$$

with an additional hard-core condition $a_{k}^{2}=0$, we can construct a nearest-neighbor interacting anyonic model, proposed recently [7]:

$$
C_{1}=H^{(1)}=\sum_{k=1}^{N} 2 n_{k} n_{k+1}+a_{k} a_{k+1}^{\dagger}+a_{k}^{\dagger} a_{k+1}, \quad n_{k} \equiv a_{k}^{\dagger} a_{k} .
$$

Operators $a_{k}^{\dagger}, a_{k}$ obey the anyonic CR at space-separated points $k>l$ :

$$
a_{k} a_{l}^{\dagger}=e^{i \theta} a_{l}^{\dagger} a_{k}, \quad a_{k} a_{l}=e^{-i \theta} a_{l} a_{k},
$$

with $\theta=0$ giving commuting bosonic and $\theta=\pi$ anticommuting fermionic relations. but behave like a fermion with the anticommutation relation $\left[a_{k}, a_{k}^{\dagger}\right]_{+}=1$, at the coinciding points, confirming the deficiency of the existing anyonic models, mentioned above.

ii) Anyonic lattice model. For a different realization of the general operator $\mathbf{p}$ satisfying BYBE:

$$
p_{12}^{(k)}=\psi_{k}, \quad p_{21}^{(k)}=\tilde{\psi}_{k}, \quad p_{11}^{(k)}=n_{k} \equiv p_{k}+\tilde{\psi}_{k} \psi_{k},
$$

together with some other conditions, but without hard-core restriction, anyonic operators $\psi_{k}$, $\tilde{\psi}_{k}, p_{k}$ can construct another quantum integrable anyonic model with next-nearest neighbor interactions and higher order nonlinearity given by the Hamiltonian

$$
C_{3}=\mathrm{H}^{(2)}=\sum_{k}\left(\tilde{\psi}_{k+1} \psi_{k-1}-\left(n_{k}+n_{k+1}\right) \tilde{\psi}_{k+1} \psi_{k}+\frac{1}{3 \Delta^{2}} n_{k}^{3}\right)
$$

Remarkably, this model gives the needed anyonic CR at the coinciding points $k$ :

$$
\psi_{k} \tilde{\psi}_{k}-e^{-i \theta} \tilde{\psi}_{k} \psi_{k}=p_{k}
$$

together with

$$
\psi_{k} \tilde{\psi}_{j}=e^{i \theta} \tilde{\psi}_{j} \psi_{k}
$$

and other similar relations at noncoinciding points $k>j$. Thus we solve here one of the existing problems of anyonic models by constructing the anyonic operator with proper CR, valid at all points.

iii) Quantum integrable NLS anyonic field model. Taking carefully the continuum limit of the lattice anyonic model (12) with $k \rightarrow x$, and the field $\psi_{k} \rightarrow A(x), \tilde{\psi}_{k} \rightarrow A^{\dagger}(x)$, we can derive a novel NLS anyonic field model

$$
\hat{H}^{(3)}=\int d x\left(A_{x}^{\dagger} A_{x}+c\left(A^{\dagger} A\right)^{2}\right)
$$


with the anyonic field operator $A(x)$ obeying the needed CR at all points, obtained from its lattice counterpart (13), (14). At the coinciding points $x \rightarrow y^{+}$we get the anyonic $\mathrm{CR}$ for the field operators as

$$
A(x) A^{\dagger}(y)-e^{i \theta} A^{\dagger}(y) A(x)=\delta(x-y)
$$

together with the anyonic relations at $x>y$ :

$$
A(x) A^{\dagger}(y)=e^{i \theta} A^{\dagger}(y) A(x), \quad A(x) A(y)=e^{-i \theta} A(y) A(x) .
$$

Clearly these anyonic operator relations can interpolate between the bosonic (at $\theta=0)$ and fermionic (at $\theta=\pi$ ) CR at all points.

We solve another outstanding problem of the anyonic models by finding the $N$-particle sector

$$
|N\rangle=\int d^{N} x \sum_{\left\{x_{l}\right\}} \Phi\left(x_{1}, x_{2}, \ldots, x_{N}\right) A^{\dagger}\left(x_{1}\right) A^{\dagger}\left(x_{2}\right) \cdots A^{\dagger}\left(x_{N}\right)|0\rangle
$$

of the NLS anyonic field model (15), which indeed gives the well known $\delta$-function anyon gas model

$$
H_{N}=-\sum_{k} \partial_{k}^{2}+c \sum_{k \neq j} \delta\left(x_{k}-x_{l}\right)
$$

linking it thus with a genuine 1D anyonic quantum field model. All the above anyonic models are obtained using the rational $R$-matrix solution. Now we switch over to the trigonometric case, presenting only our main result, with all details intended for a separate report [15].

\subsection{Trigonometric class of anyonic models}

We consider now the trigonometric quantum $R$-matrix (6), (8) related to the $x x z$ spin- $\frac{1}{2}$ chain and a corresponding trigonometric modification of the Lax operator (11) through abstract anyonic $q$-oscillator operator $\boldsymbol{\tau} \equiv\left(\tau_{12}, \tau_{21}, \tau_{a}^{ \pm}, a=1,2\right)$, keeping however the same braiding matrix $Z$ (9). Following the scheme for constructing the integrable models starting from the Lax operator as discussed above and using different concrete realizations of the operator $\tau$, we generate a trigonometric class of anyonic lattice and field models, with a deformation parameter

$q=e^{i \alpha}$, in addition to the anyonic parameter $\theta$. Different forms of $q$-anyonic algebras can be derived from the BYBE and the BR relations for different realizations of $\boldsymbol{\tau}$.

iv) $\boldsymbol{q}$-anyonic model. Using a realization through quantum anyonic operators:

$$
\tau_{1}^{ \pm}=q^{\mp N}, \quad \tau_{2}^{ \pm}=q^{ \pm N} e^{i \theta N}, \quad \tau_{12}=\tilde{\phi}, \quad \tau_{21}=\phi,
$$

we get from BYBE (4) and (5) an anyonic q-oscillator model with CR:

$$
\phi_{k} \tilde{\phi}_{k}-e^{i \theta} \tilde{\phi}_{k} \phi_{k}=e^{i \theta N_{k}} \cos 2 \alpha N_{k}
$$

at the coinciding points and

$$
\phi_{k} \tilde{\phi}_{j}=e^{i \theta} \tilde{\phi}_{j} \phi_{k}
$$

at the space-separated points $k>j$, showing that we can generate through our scheme the anyonic algebra relations both at the coinciding and noncoinciding points, even for the quantum deformation, which was another aim we started with. Now we intend to find the corresponding integrable quantum field model, which is our other major aim. Therefore relegating further 
details of this anyonic $q$-oscillator model [15], we go to the continuum limit of this model and present below another anyonic quantum integrable field model.

v) Derivative NLS anyonic field model. At the field limit by taking lattice constant $\Delta \rightarrow 0$, the anyonic $q$-oscillator would reduce to an anyonic field operator: $\phi_{k} \rightarrow D(x)$, to derive from the $q$-anyonic lattice model a quantum integrable derivative NLS anyonic field model

$$
\hat{H}^{(5)}=\int d x\left(D_{x}^{\dagger} D_{x}+2 i \kappa\left(D^{\dagger}\right)^{2} D D_{x}\right)
$$

with the anyonic field operator satisfying at $x \rightarrow y^{+}$the $\mathrm{CR}$

$$
D(x) D^{\dagger}(y)-e^{i \theta} D^{\dagger}(y) D(x)=\kappa \delta(x-y)
$$

and

$$
D(x) D^{\dagger}(y)=e^{i \theta} D^{\dagger}(y) D(x)
$$

at $x>y$. Interestingly, even at the field limit the important anyonic operator relations remain valid at the coinciding (16) as well as at the noncoinciding (17) points. We find that, this anyonic DNLS field model gives at its $N$-particle sector $|N\rangle$, the recently proposed derivative $\delta$-function anyon gas model

$$
H_{N}^{d}=-\sum_{k} \partial_{k}^{2}+i \kappa \sum_{k \neq j} \delta\left(x_{k}-x_{l}\right)\left(\partial_{x_{k}}+\partial_{x_{l}}\right)
$$

establishing thus the missing link of this anyon gas to a quantum integrable anyonic field model, discovering which was our final aim.

We have constructed here from the BYBE a series of anyonic and $q$-anyonic models, namely i) nearest-neighbor hard-core anyonic, ii) next-nearest-neighbor higher nonlinear lattice anyonic and iii) quantum NLS anyonic field models from the rational class and iv) anyonic $q$-oscillator and v) anyonic DNLS quantum field models from the trigonometric class. We emphasize that, except the first one i) all other anyonic models are new, presented here for the first time and are quantum integrable models, solvable by the algebraic Bethe ansatz. Postponing the relevant details of the Bethe ansatz formulation to [15] we just mention that, it goes pretty close to the standard formulation for the ultralocal models [16]. The anyonic contribution entering through the braiding matrix $Z$, due to its simple form considered here, does not create much difficulty and follow the line of [13], though individual nonultralocal model presented here would have its specific problem and solution.

\section{Concluding remarks}

We have constructed two classes of 1D anyonic models, rational and trigonometric, in a systematic way starting from the braided YBE. The known as well as new anyonic models, that we have constructed, are all quantum integrable and exactly solvable by the algebraic Bethe Ansatz. Among the new models a next-nearest neighbor interacting lattice anyonic model and a nonlinear Schrödinger anyonic quantum field model belong to the rational class. Among the models belonging to the trigonometric class we have discovered an anyonic $q$-oscillator lattice model and a derivative NLS anyonic field model.

Remarkably, the NLS anyonic quantum field model at its $N$-particle sector recovers the $\delta$-function anyon gas, while the derivative NLS anyonic field model is linked to the recently proposed derivative $\delta$ anyon gas. Thus we find the quantum integrable field models corresponding to the known anyon gas models, solving an outstanding problem. 
The anyonic models we have constructing here exhibit proper anyonic $\mathrm{CR}$ at all points, resolving another problem of the existing models.

Investigating further the nonultralocal BYBE in the trigonometric case, we have obtained recently a new kind of anyonic quantum group Hopf algebra, with an additional deformation given by the anyonic parameter. We hope that, this two-parameter quantum algebra with explicit nonultralocal nature, which is still under study and will be reported in time [15], would be a significant addition in the knowledge of quantum algebras.

Another promising line of research would be to find nonabelian realizations of the integrable 1D anyonic models exploiting the BYBE, which might shed light to the nonabelian anyonic models, importance of which is growing in recent years [3].

\section{References}

[1] Wilczek F., Magnetic flux, angular momentum and statistics, Phys. Rev. Lett. 48 (1982), 1144-1146.

[2] Camino F.F., Zhou W., Goldman V.J., Realization of a Laughlin quasiparticle interferometer: observation of fractional statistics, Phys. Rev. B $\mathbf{7 2}$ (2005), 075342, 8 pages, cond-mat/0502406.

Kim E., Lawer M., Vishweshwara S., Fradkin E., Signature of fractional statistics in noise experiments in quantum Hall fluids, Phys. Rev. Lett. 95 (2005), 176402, 4 pages, cond-mat/0507428.

[3] Kitaev A.Yu., Fault-tolerant quantum computation by anyons, Ann. Physics 303 (2003), 2-30, quant-ph/9707021.

Kitaev A.Yu., Anyons in an exactly solved model and beyond, Ann. Physics 321 (2006), 2-111, cond-mat/0506438.

Trebst S., Troyer M., Wang Z., Ludwig A.W., A short introduction to Fibonacci anyon models, Progr. Theoret. Phys. Suppl. 176 (2008), 384-407, arXiv:0902.3275.

Nayak C., Simon S.H., Stern A., Freedman M., Das Sarma S., Non-abelian anyons and topological quantum computation, Rev. Modern Phys. 80 (2008), 1083-1159, arXiv:0707.1889.

[4] Pâtu O.I., Korepin V.E., Averin D.V., Correlation functions of one-dimensional Lieb-Liniger anyons, J. Phys. A: Math. Theor. 40 (2007), 14963-14984, arXiv:0707.4520.

[5] Kundu A., Exact solution of double $\delta$-function Bose gas through an interacting anyon gas, Phys. Rev. Lett. 83 (1999), 1275-1278, hep-th/9811247.

[6] Batchelor M.T., Guan X.-W., Kundu A., One-dimensional anyons with competing $\delta$-function and derivative $\delta$-function potentials, J. Phys. A: Math. Theor. 41 (2008), 352002, 8 pages, arXiv:0805.1770.

[7] Batchelor M.T., Foerster A., Guan X.-W., Links J., Zhou H.-Q., The quantum inverse scattering method with anyonic grading, J. Phys. A: Math. Theor. 41 (2008), 465201, 13 pages, arXiv:0807.3197.

[8] Batchelor M.T., Guan X.-W., Oelkers N., One-dimensional interacting anyon gas: low energy properties and Haldane exclusion statistics, Phys. Rev. Lett. 96 (2006), 210402, 4 pages, cond-mat/0603643.

Girardeau M.D., Anyon-fermion mapping and applications to ultracold gasses in tight waveguides, Phys. Rev. Lett. 97 (2006), 100402, 4 pages, cond-mat/0604357.

Averin D.V., Nesteroff J.A., Coulomb blockade of anyons in quantum antidots, Phys. Rev. Lett. 99 (2007), 096801, 4 pages, arXiv:0704.0439.

Batchelor M.T., Guan X.-W., He J.-S., The Bethe ansatz for one-dimensional interacting anyons, J. Stat. Mech. Theory Exp. 2007 (2007), P03007, 19 pages, cond-mat/0611450.

Calabrese P., Mintchev M., Correlation functions of one-dimensionalanyonic fluids, Phys. Rev. B 75 (2007), 233104, 4 pages, cond-mat/0703117.

Santachiara R., Increasing of entangement entropy from pure to random quantum critical chains, J. Stat. Mech. Theory Exp. 2006 (2006), L06002, 8 pages, cond-mat/0602527.

Pâtu O.I., Korepin V.E., Averin D.V., One-dimensional impenetrable anyons in thermal equillibrium. I. Anyonic generalizations of Lenard's formula, J. Phys. A: Math. Theor. 41 (2008), 145006, 15 pages, arXiv:0801.4397.

Pâtu O.I., Korepin V.E., Averin D.V., One-dimensional impenetrable anyons in thermal equillibrium. II. Determinant represenation for the dynamic correlation functions, J. Phys. A: Math. Theor. 41 (2008), 255205, 19 pages, arXiv:0803.0750.

del Campo A., Fermionization and bosonization of expanding one-dimensional anyonic fluids, Phys. Rev. A 78 (2008), 045602, 4 pages, arXiv:0805.3786.

[9] Keilmann T., Lanzmich S., McCulloch I., Roncaglia M., Statistically induced phase transitions: turning bosons smoothly via anyons into fermions, arXiv:1009.2036. 
[10] Lieb E.H., Liniger W., Exact analysis of an interacting Bose gas. I. The general solution and the ground state, Phys. Rev. 130 (1963), 1605-1616.

[11] Shnirman A.G., Malomed B.A., Ben-Jacob E., Nonperturbative studies of a quantum higher order nonlinear Schrödinger model using the Bethe ansatz, Phys. Rev. A 50 (1994), 3453-3463.

[12] Hlavatý L., Kundu A., Quantum integrability of non-ultralocal models through Baxterisation of quantised braided algebra, Internat. J. Modern Phys. A 11 (1996), 2143-2165, hep-th/9406215.

[13] Kundu A., Exact Bethe ansatz solution of non-ultralocal quantum mKdV model, Modern Phys. Lett. A 10 (1995), 2955-2966, hep-th/9510131.

[14] Faddeev L.D., Quantum completely integrable models in field theory, in Mathematical Physics Reviews, Soviet Sci. Rev. Sect. C: Math. Phys. Rev., Harwood Academic, Chur, 1980, Vol. 1, 107-155.

[15] Kundu A., Exact solution of anyonic NLS quantum field model, in preparation.

Kundu A., Novel nonultralocal quantum group and exactly solvable anyonic DNLS quantum field model, in preparation.

[16] Korepin V.E., Bogoliubov N.M., Izergin A.G., Quantum inverse scattering method and correlation functions, Cambridge Monographs on Mathematical Physics, Cambridge University Press, Cambridge, 1993. 